\title{
METODOLOGÍA PARA LA MODELACIÓN DISTRIBUIDA DE LA ESCORRENTÍA SUPERFICIAL Y LA DELIMITACIÓN DE ZONAS INUNDABLES EN RAMBLAS Y RÍOS-RAMBLA MEDITERRÁNEOS
}

\author{
Alejandro Triviño Pérez \\ Instituto Universitario de Geografía \\ Universidad de Alicante \\ Sergio Ortiz Rojas \\ Departamento de Lenguajes y Sistemas Informáticos \\ Universidad de Alicante
}

\section{RESUMEN}

Las ramblas y ríos-rambla mediterráneos se caracterizan por tener una escorrentía de carácter torrencial que con frecuencia provoca avenidas e inundaciones. En este artículo se propone un método para estimar la propagación de caudales y niveles de agua en este tipo de cuencas durante una precipitación de fuerte intensidad horaria. La metodología está basada en un modelo hidrológico distribuido construido con ayuda de un Sistema de Información Geográfica (SIG) para el pre- y postprocesado de las variables hidrológicas. Sus resultados aportan criterios rigurosos para delimitar las zonas inundables y elaborar una cartografía de calidad sobre riesgos de inundación.

Palabras clave: Ramblas, ríos-rambla, escorrentía superficial, modelos hidrológicos, Sistemas de Información Geográfica (SIG).

\begin{abstract}
Methodology for spatially distributed modeling of surface runoff and floodplain's landscape in mediterranean torrential streams. Many of the Mediterranean basin's rapid streams are characterised by torrential runoff and are known for causing flash floods. In this paper, a method is proposed to estimate watershed flow continuity and water surface elevations during turbulent stormy events. The methodology is based on a spatially distributed hydrological model that uses a Geographical Information System (GIS) to pre- and post- process hydrological data. The model's outputs provide rigorous criteria for mapping the floodplain's landscape.
\end{abstract}

Key words: Torrential streams, surface runoff, hydrological models, GIS. 


\section{Introducción}

Las manifestaciones de carácter hidrológico e hidráulico que se originan en las cuencas de drenaje constituyen uno de los sistemas geomorfológicos más destacados que operan en la superficie terrestre (López Bermúdez, 1992). Las cuencas actúan como sistemas de procesos y respuestas en donde la escorrentía superficial es su consecuencia más evidente, además de la responsable de catástrofes y desastres naturales, y de numerosos daños relacionados con las avenidas e inundaciones. Los ámbitos geográficos con climas semiáridos son un caso particular muy llamativo en donde la circulación hídrica acontece como respuesta a precipitaciones que superan unos determinados umbrales de intensidad y magnitud (Mateu, 1989). En ellos, la distribución anual de las lluvias regula el régimen de los aparatos fluviales provocando variaciones estacionales en los caudales y configurando una red hidrológica que en el mediterráneo está formada mayoritariamente por ramblas y ríosrambla (Gil, 1993). Las ramblas son corrientes fluviales efímeras en las que sólo circula agua durante escasos días al año y siempre como resultado de precipitaciones de fuerte intensidad horaria. Registran módulos absolutos modestos, caudales relativos ínfimos, y, en consecuencia, una irregularidad interanual tendente a infinito. Los ríos-rambla como indica su nombre ocupan un puesto intermedio entre los ríos y las ramblas. Sus cuencas tienen mayor extensión que las anteriores y suelen estar mejor alimentadas en cabecera debilitándose conforme se avanza hacia la desembocadura. Sus módulos son inferiores a 2 $\mathrm{m}^{3} / \mathrm{s}$ e incluso habitualmente registran valores menores a $1 \mathrm{~m}^{3} / \mathrm{s}$ cuando no reciben aportaciones extraordinarias (p. ej. manantiales). Esta descripción aunque breve es lo suficientemente ilustrativa como para inferir que en estos cursos fluviales hablar de actividad hidrológica es tanto como referirse a las crecidas provocadas por lluvias torrenciales (Mateu, 1989).

En ramblas y ríos-rambla mediterráneos la escorrentía superficial es la respuesta hidrológica a las precipitaciones de fuerte intensidad horaria. Entendidas como sistemas abiertos en donde sus componentes intercambian materia y energía con el entorno (López Bermúdez, 1992), el enfoque sistémico nos aporta una visión práctica y funcional que ayuda a centrar el análisis en las manifestaciones hidrológicas más importantes, simplificando el procedimiento para identificar los factores que de manera más relevante intervienen en el proceso de conversión lluvia-caudal. De este modo, la construcción teórica de un sistema hidrológico orientado al estudio de la escorrentía entraña la determinación de los elementos que lo componen y la caracterización del conjunto de principios y reglas que racionalmente los conectan. Este esquema mejora con creces la comprensión de la escorrentía facilitando su abstracción en modelos matemáticos capaces de simular su comportamiento y de cartografiar su distribución en el espacio. En relación con este último aspecto, debemos tener presente que a diferencia de los modelos agregados, las modelaciones distribuidas persiguen la simulación completa del fenómeno, lo que significa analizar el calado de las aguas, la velocidad de éstas y la duración de la inundación considerando su distribución en el espacio. Estas magnitudes proporcionan una información esencial para delimitar las zonas inundables de una cuenca, y, evidentemente, para cartografiar el riesgo de inundación de un territorio en función de las consideraciones realizadas para nuestro país por la Directriz Básica de Protección Civil para este tipo de riesgos (BOE del 14 de febrero de 1995).

La modelación distribuida a la que hacemos referencia se ve dificultada por la complejidad y dinamismo que caracteriza a la escorrentía superficial, pues precisa de ingentes cantidades de datos que sólo pueden ser gestionados eficientemente por dispositivos electrónicos dotados con una memoria de gran capacidad y de métodos para el tratamiento de 
la información. Los modelos hidrológicos, al igual que los hidráulicos, implementados en un ordenador, cumplen estas expectativas conformando una vía de trabajo que incrementa la potencia de las operaciones de cálculo y el nivel de detalle de la cartografía. Con todo, las simulaciones hidrológicas todavía cuentan con serias limitaciones operativas, especialmente en lo que se refiere al tamaño de los datos, siendo inevitable realizar una simplificación de la realidad mediante la selección de las variables que de manera más significativa participan en el sistema. Estos inconvenientes de índole técnica no menoscaban la utilidad de los modelos, ya que empleando sistemas de información capaces de generar funciones específicas para el análisis hidrológico, se ha conseguido mejorar la calidad de sus resultados al dotarlos con información relativa a su localización espacial. Los Sistemas de Información Geográfica (SIG) son un excelente ejemplo de ello, constituyen un instrumento de trabajo que proporciona un medio adecuado para responder a cuestiones relacionadas con la componente espacial de la escorrentía. Estos sistemas facilitan el tratamiento de la información hidrológica gracias a que incluyen procedimientos diseñados para realizar la captura, almacenamiento, manipulación, análisis, modelación y presentación de datos georreferenciados (NCGIA, 1990). Por ello, los SIG habitualmente se utilizan para suministrar al modelo las características topológicas de la red hidrológica, la forma de las secciones transversales de los cauces, los recorridos de los flujos de agua, los coeficientes de Manning, etc. La modelación distribuida de la escorrentía superficial con ayuda de los SIG acelera su desarrollo y aplicación pudiendo actuar a modo de plataforma para la experimentación rápida de nuevas ideas y conceptos.

\section{La modelación de fenómenos hidráulicos e hidrológicos}

Simular el comportamiento de una cuenca hidrológica con la ayuda de un modelo matemático cobra sentido cuando la información obtenida con el mismo resulta imprescindible para lograr un objetivo difícilmente alcanzable por otros medios menos costosos. Un argumento que justifica el estudio hidrogeomorfológico de las ramblas y ríos-rambla mediterráneos es el análisis del riesgo de inundación, habida cuenta de la gran superficie de usos residenciales y actividades económicas que se concentran en los márgenes y llanos de inundación de estos cauces. Los modelos facilitan la manipulación de las variables que intervienen en el proceso de conversión lluvia-caudal, aportando datos sobre la distribución espacial de la escorrentía superficial y mejorando la calidad de la cartografía temática sobre zonas inundables y zonas con riesgo de inundación. Hay, por tanto, razones para intentar superar los desafíos que plantea la modelación distribuida de fenómenos hidrológicos e hidráulicos, no en vano lo que se pretende es aumentar la eficacia de los instrumentos de ordenación territorial, incluido los urbanísticos, y de los planes de emergencia ante inundaciones.

Los modelos matemáticos encargados de simular el comportamiento de las cuencas de drenaje se denominan «modelos hidrológicos» y «modelos hidráulicos». Ahora bien, ¿a qué hacen referencia cada uno de ellos? Con frecuencia, la literatura existente sobre el tema no ayuda a responder de manera clara y directa a esta cuestión, puesto que las dos denominaciones habitualmente han sido utilizadas como sinónimas creando una cierta confusión sobre cuales son sus verdaderos objetos de estudio. Para evitar equívocos sobre el contenido de los modelos hidrológicos de un lado, y los hidráulicos de otro, es recomendable precisar sus límites conceptuales utilizando un criterio amplio y sencillo donde el modelo se corresponda con el contenido de la palabra que le califica. Trasladado al asunto que nos atañe esta función vendría dada por los adjetivos hidrológico e hidráulico, y por ende sus objetos de estudio residirían en la hidrología y en la hidráulica respectivamente. 
Desde este punto de vista, el contenido de estos modelos tiene que corresponderse con las materias que ocupan a estas dos ciencias, circunscribiéndose a las siguientes definiciones:

- La hidrología es la encargada del estudio de las características físicas y químicas de las aguas continentales y marinas, de sus movimientos y de las transformaciones que experimentan a lo largo del ciclo hidrológico (Galiana, 1987).

- El objeto de estudio de la hidráulica es el equilibrio y el movimiento de los fluidos, es decir, el comportamiento mecánico del agua en reposo y en movimiento en conducciones abiertas y cerradas, entendiendo como tales los cauces, canales y tuberías, así como estructuras y dispositivos hidráulicos por los que fluye el agua y medios permeables por los que se filtra (López Andrés, 1997).

Si examinamos detenidamente estas definiciones llegamos a la conclusión de que los modelos hidrológicos pueden centrarse en una, en varias o en todas las secuencias que componen el ciclo hidrológico. Esto es, en las sucesivas etapas por las que el agua pasa de la atmósfera a la corteza terrestre y de ésta de nuevo a la atmósfera implicando: precipitación, acumulación, infiltración, circulación, evaporación, transpiración y condensación (López Bermúdez, 1992). Estas fases presentan un dinamismo inherente al propio ciclo que posibilita la circulación hídrica en la superficie terrestre y convierte el desplazamiento de caudales en conducciones abiertas en el punto de encuentro de la hidráulica y la hidrología. De este modo, para comprender la escorrentía hay que recurrir inexcusablemente a los principios que rigen la mecánica de fluidos, aunque sin olvidar que el escurrimiento es, tan sólo, uno de los múltiples procesos que se producen en las cuencas de drenaje y que se resuelven en el seno del ciclo hidrológico. Esta visión sistémica de los hechos posiciona a la hidráulica dentro del mencionado ciclo, actuando como ciencia subsidiaria del conocimiento hidrológico cuando es aplicada al estudio del equilibrio y el movimiento del agua en los cauces.

La fuerte conexión entre los modelos hidrológicos y los hidráulicos no oculta las diferencias existentes entre ellos, por lo que tampoco legitima el hecho de que estas dos denominaciones puedan ser utilizadas indistintamente confundiendo sus contenidos. Son numerosos los ejemplos que desacertadamente suponen que un modelo hidrológico estudia los procesos que no son analizados por el hidráulico. Esta concepción es excluyente y obliga a implementar por separado ambos modelos para completar el ciclo hidrológico. En otras ocasiones se omite la denominación de hidrológico y se asigna al modelo hidráulico todas las funciones necesarias para simular la escorrentía, es decir, se le atribuye el estudio de factores que como los climáticos exceden a sus competencias. Más exacto es hablar de modelos hidrológicos integrados por modelos hidráulicos. En estos casos, los primeros dan nombre a todo el procedimiento, mientras que los segundos actúan como complemento centrándose exclusivamente en la simulación del comportamiento del agua en la superficie terrestre. Estas circunstancias exigen la descripción aunque sea muy brevemente de estos dos tipos de modelos.

\subsection{Modelos hidráulicos}

Estos modelos matemáticos han adquirido gran difusión en muchos campos de trabajo relacionados con la hidrología, siendo recomendable realizar alguna valoración sobre ellos para comprender mejor su finalidad, funcionamiento y resultados. Ante esta tesitura, ineludiblemente debemos hacer referencia a la labor desarrollada por el Hydrologic Engineering Center(HEC) del United States Army Corps of Engineers, un centro que ha 
diseñado los modelos hidráulicos con mayor reconocimiento y aceptación internacional. A modo ilustrativo podemos mencionar el célebre modelo unidimensional HEC-2. Sus antecedentes datan de 1966 con la aparición del programa «Backwater Any Cross Section» y en 1995 fue reemplazado por el HEC-RAS «River Analysis System» cuya versión más reciente es de abril de 2004. En España, una muestra interesante de la aplicación del HEC-2 son los mapas de riesgo de inundación y riesgos asociados que el Servei Geològic de Catalunya elabora desde la década de los 90 .

La concepción de todos los modelos hidráulicos es parecida por lo que una breve explicación del HEC-RAS puede ayudarnos a comprender mejor las ventajas e inconvenientes de su aplicación. Este paquete informático integra varios programas de análisis hidráulico en donde el usuario se comunica con el sistema a través de una interfaz gráfica. El objetivo primordial del modelo es obtener la altura del agua en ríos con regímenes permanentes o de flujos discontinuos calculando el área mojada de las secciones transversales, si bien su arquitectura abierta también admite otras posibilidades relacionadas con la acción geomorfológica de la escorrentía (p. ej. la denudación fluvial). La realización en el HEC-RAS de un proyecto ordinario orientado al análisis de zonas inundables, requiere información relacionada con la fisiografía del aparato fluvial y los registros foronómicos, debiéndose sistematizar en los siguientes apartados: datos geométricos del cauce, flujos de agua y especificaciones técnicas de la simulación.

Los datos geométricos hacen referencia al aspecto de la sección transversal del cauce, pieza clave del modelo porque a partir de ella se realizan todos los cálculos (vid. Figura 1). En el HEC-RAS las secciones transversales se introducen manualmente -interpretando la cartografía topográfica o con mediciones directas en el campo con aparatos GPS - ubicándolas una tras otra a lo largo del eje del cauce, teniendo en cuenta que deben ser perpendiculares al mismo y estar poco distantes entre sí. Una extensión para SIG, el HEC-GeoRAS, facilita esta tarea al permitir obtenerlas automáticamente sobre un Modelo Digital de Elevaciones (MDE). Las secciones, por la naturaleza de la información que contienen,

FIgURA 1. Sección transversal de un río realizada en el HEC-RAS (Tate, 1999).

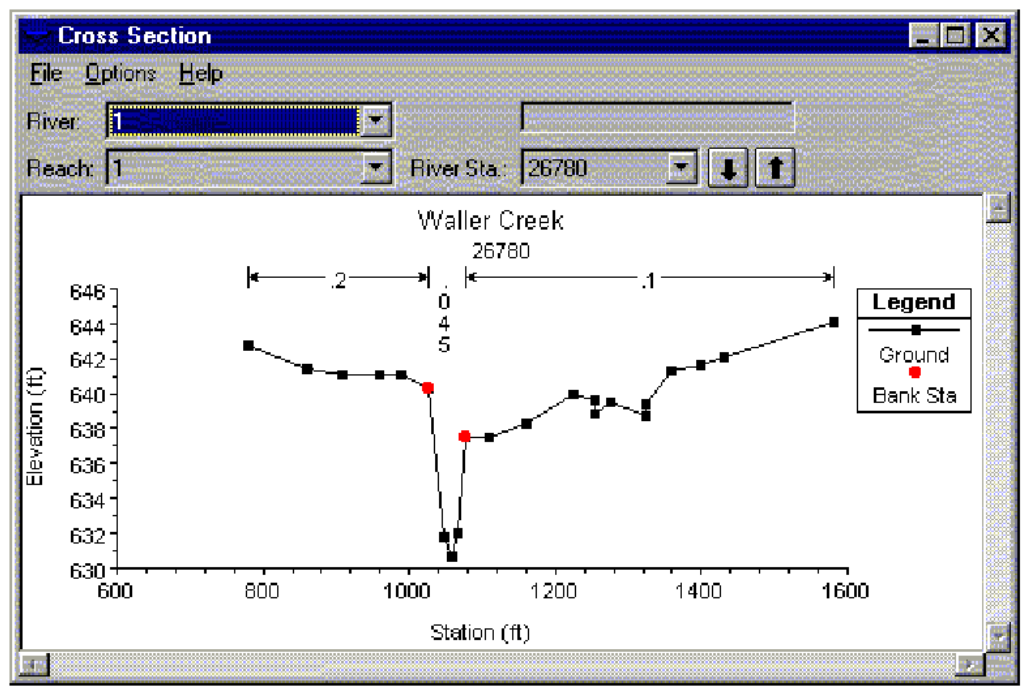


conviene ubicarlas en lugares donde se producen variaciones significativas en las condiciones hidráulicas, siendo el caso de las zonas con pérdida de carga y espacios con importantes cambios en la pendiente, en el coeficiente de rugosidad de Manning o en el perfil, e incluso se debe considerar la presencia de obras civiles (Andrysiak, 2000). Otro nivel de información al que nos hemos referido con anterioridad son los flujos de agua, es decir, los caudales de descarga registrados en distintos puntos del cauce. Estos datos se obtienen de las estaciones de aforos o bien estimándolos con la simulación de una tormenta de diseño en un modelo hidrológico. Respecto a este particular, debemos indicar que el HEC-RAS esta preparado para importarlos directamente del modelo hidrológico HEC-HMS.

Los resultados generados por el HEC-RAS pueden ser exportados a un SIG gracias a un archivo de intercambio que contiene la localización de las secciones transversales, sus respectivos calados y los polígonos que describen las zonas inundables (Maidment y Djokic, 2000). Sin embargo no todo es positivo, modelos hidráulicos como el descrito realizan los cálculos basándose en las secciones del cauce, lo cual dificulta la representación cartográfica de los resultados a escala de cuenca. Estos modelos tienen una aplicación restringida, pues se circunscriben al lecho fluvial, omitiendo el comportamiento hídrico del resto de la cuenca, y, consecuentemente, las relaciones de vecindad y la continuidad espacial entre las zonas ribereñas y las más alejadas al cauce. Así, la difusión de los modelos hidráulicos es fácil de constatar en determinados proyectos de ingeniería, pero no tanto en estudios territoriales, pues en estos últimos lo que prima es la facilidad para interpretar los resultados de manera amplia en el espacio geográfico, es decir, se valora la producción cartográfica de la información hidrológica a escala de cuenca.

\subsection{Modelos hidrológicos}

Los modelos hidrológicos entendidos como una aproximación al funcionamiento real del ciclo del agua en una cuenca vertiente se pueden clasificar en físicos y abstractos. A su vez los modelos abstractos de acuerdo con la aleatoriedad de las variables empleadas pueden ser estocásticos o deterministas. En este artículo nos centramos en esta última tipología, en concreto, en los modelos de parámetros distribuidos. A diferencia de los modelos de parámetros agregados, los distribuidos prestan mayor atención a la distribución espacial de los datos en la cuenca, pero no sólo a los asociados con el escurrimiento superficial del agua sino que también a factores climáticos como las precipitaciones. Con ellos se supera la escala de trabajo habitual de los modelos hidráulicos, los cuales se ciñen casi exclusivamente al cauce como consecuencia de la utilización de las secciones transversales de los ríos para realizar los cálculos. Los modelos hidrológicos distribuidos se aproximan mucho más a la realidad al aceptar que la escorrentía es una respuesta hidrológica continua en el espacio y en el tiempo que afecta de distinta manera a toda la superficie de la cuenca. Se reafirma el hecho de que es importante poder consultar la información sobre lo que sucede en cualquier punto de la cuenca. Así, estos modelos simulan la escorrentía en un lugar concreto considerando la influencia del espacio adyacente, es decir, las zonas que directa o indirectamente intervienen en él vertiendo y drenando el agua precipitada.

Al igual que hemos realizado en el apartado anterior explicando los modelos hidráulicos en función del HEC-RAS, en éste podemos ilustrar los modelos hidrológicos deterministas de parámetros distribuidos sirviéndonos del HEC-HMS «Hydrologic Modeling System». Este bienquisto programa proporciona a sus usuarios varias opciones para simular el comportamiento de la escorrentía superficial. Entre ellas se ha incluido el método del 
FIGURA 2. Especificaciones técnicas realizadas en el modelo hidrológico HEC-HMS (Andrysiak, 2000).

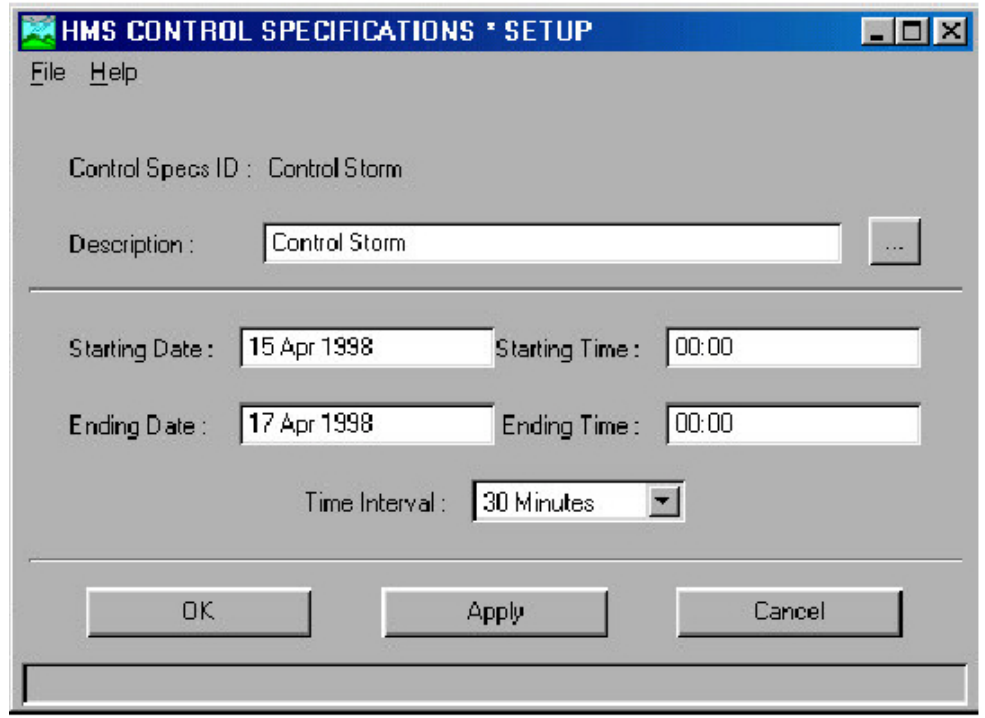

hidrograma unitario (HU) para la obtención del caudal punta y algoritmos para la asignación de rutas a los caudales, esto último con la particularidad de que puede ser implementado en un formato ráster legible por los SIG. Para trabajar con el HEC-HMS, además de las especificaciones técnicas de la simulación (vid. Figura 2), son imprescindibles los datos de las precipitaciones y de las características morfológicas de la cuenca. Puesto que en este tipo de modelos se coloca el énfasis en la distribución geográfica de la información y sus variaciones en el tiempo, resulta crucial evitar errores relacionados con la componente espacial y las series temporales de los datos, especialmente en lo que concierne al medio físico-ecológico, pues se ha contrastado empíricamente su influencia en la continuidad del fenómeno lo que repercute en la calidad de los resultados de la simulación. Esto también sucede con los errores de etiquetado que afectan a la componente temática de los usos del suelo (formaciones vegetales incluidas), situándose el grado de exactitud entre el 60 y el $85 \%$ dependiendo de la complejidad del área de estudio (Choudhry y Morad, 1998).

El HEC-HMS se vale de los MDE para representar el elemento abiótico del paisaje de la cuenca, conviniendo emplear en su construcción la red hidrológica para definir con mayor detalle las principales líneas de flujo del territorio drenado. El modelo también asume la influencia de las actividades humanas en la escorrentía empleando el método del número de curva creado por el Soil Conservation Service (SCS) del Department of Agriculture de los EE.UU. (1985). Este método estima la fracción de agua que no se infiltra de acuerdo con los usos del suelo, su textura y la pendiente topográfica. También son útiles los parámetros de la velocidad de la corriente y la propagación de la onda de crecida (método de Muskingum-Cunge). En cuanto a las lluvias, son necesarias series cronológicas que nos permitan calcular la precipitación máxima según distintos períodos de retorno. Con ellas se pueden diseñar tormentas hipotéticas y estimar razonadamente la cantidad e 
intensidad de lluvia por unidad de tiempo en cada punto de la cuenca. La distribución espacial y temporal de las precipitaciones sirve además para inquirir el desplazamiento de las células convectivas y establecer una correlación entre lluvia y escorrentía de acuerdo con las características del medio.

Con todos estos datos el HEC-HMS resuelve el problema de la conversión lluviacaudal estimando previamente las pérdidas producidas por la infiltración. Esta función se puede utilizar para simulaciones continuas en el tiempo siempre y cuando se especifique la humedad y la cantidad de agua que es capaz de retener el suelo antes de saturarse (Andrysiak, 2000). Con esta información la escorrentía se puede estimar de dos formas: acumulada, cuando los resultados se obtienen para toda la cuenca; y distribuida, para el caso de datos organizados en una retícula, o sea, en formato ráster. En este último la precipitación, el agua infiltrada y el grado de saturación del suelo se obtienen para cada una de las celdas que componen la retícula. Al agua que queda en la superficie se le asignan rutas para dirigirla a los colectores de evacuación de la cuenca simulando la circulación de caudales.

La consideración de todas las fases que integran el ciclo hidrológico exige al modelo una formidable capacidad analítica para establecer las conexiones y el comportamiento de las variables naturales y antrópicas que participan en la escorrentía. Estas variables son muy dinámicas y durante un episodio de lluvias de fuerte intensidad horaria experimentan constantes modificaciones en el espacio y en el tiempo. Esto complica enormemente los cálculos debido al ingente volumen de información que debe ser procesado, siendo aconsejable simplificarlas para poder trabajar con una cierta comodidad en plataformas de bajo coste (p. ej. estaciones de trabajo). Para evitar que tal cantidad de información se convierta en un criptograma de imposible lectura, hay que utilizar sistemas de información capaces de gestionar, analizar y visualizar datos espaciales. El hecho de que modelos inicialmente pensados para el diseño de obras civiles también sirvan para prevenir riesgos de inundación, obliga a buscar soluciones que mejoren la representación cartográfica de sus resultados. La integración de estos modelos en los SIG elimina estas carencias e incluso mejora la aprehensión de los procesos y respuestas que se dan en las cuencas.

\section{Metodología para la modelación distribuida de ramblas y ríos-rambla mediterráneos}

La metodología que proponemos en este artículo tiene como objetivo estimar la escorrentía superficial en cuencas de ramblas y ríos-rambla mediterráneos, para de este modo disponer de criterios rigurosos que nos permitan delimitar las zonas inundables y elaborar una cartografía de calidad sobre riesgos de inundación. Así, el análisis de la escorrentía y de sus consecuencias, entiéndase principalmente avenidas e inundaciones, lo hemos basado en un modelo hidrológico pensado para ser aplicado en ámbitos semiáridos. Habitualmente para trabajar con este tipo de cuencas, en general pequeñas y sin estaciones de aforos, se emplean modelos agregados que partiendo de unas condiciones geomorfológicas y climáticas fácilmente observables, consideran que la lluvia es uniforme y las variables hidrológicas globales y constantes durante el hidrometeoro. Estos modelos son útiles para obtener el valor de los parámetros de diseño necesarios en numerosos proyectos de ingeniería (Martínez et al., 2000), pero son claramente insuficientes para fijar con precisión las áreas inundables de un curso fluvial. Por ello, para el estudio de ramblas y ríos-rambla con escorrentías torrenciales parece más acertado implementar modelos hidrológicos distribuidos porque utilizando la distribución espacial y la variación temporal de las precipitaciones se aproximan mucho más al funcionamiento real de las cuencas hidrológicas.

El modelo hidrológico distribuido simula la propagación de caudales y niveles de agua en ramblas y ríos-rambla, considerando las dos dimensiones del plano horizontal y repro- 
duciendo las siguientes etapas del ciclo hidrológico: precipitación, acumulación, infiltración y circulación. La complejidad de los procesos y respuestas que se desencadenan en estos sistemas geomorfológicos nos obligan a emplear datos de origen diverso. La mayor parte responden a cuestiones de tipo espacial y pueden ser eficazmente gestionados por un SIG. Las operaciones para estimar la circulación hídrica descansan en un algoritmo de simulación especialmente diseñado para respetar la integridad de los datos en cuanto a su componente espacial y temporal se refiere. Se ha implementado un algoritmo específico debido a que modelos hidrológicos desarrollados en entornos SIG como el HEC-HMS (denominación HEC-GeoHMS), AGNPS (Agricultural Non-Point Source Pollution Model), SWAT (Soil and Water Assessment Tool), MIKE-SHE (Danish Hydraulic Institute), KINEROS (Kinematic Runoff and Erosion Model), TOPMODEL (Catchment water discharge and soil water o ANSWERS (Areal Non-point Source Watershed Environmental Response Simulation ), no se adaptan con facilidad al régimen torrencial que caracteriza a los pequeños aparatos fluviales mediterráneos. Así, el algoritmo empleado es conceptual y matemáticamente más sencillo que otros en cuanto a que simplifica el número de variables requeridas y los cálculos sobre dinámica de fluidos, aunque se ajusta con mayor precisión a la realidad. Para aplicar este modelo es preceptivo desarrollar una serie de fases que le dan cuerpo y favorecen el cumplimiento de sus objetivos. La temática abordada por cada una de las fases se corresponde con los componentes del modelo, que siguiendo el esquema de Martínez et al. (2000) deben tratar tres cuestiones básicas:

- Fase I. Tratamiento de la precipitación.

- Fase II. Síntesis de la lluvia efectiva.

- Fase III. Simulación de la escorrentía superficial.

En esta metodología, los SIG se utilizan para el pre- y postprocesado de la información geográfica, orientándose principalmente a la edición de cartografía y discretización de variables hidrológicas. Precisamente, Maidment (1993) definió varias posibilidades en el uso combinado de estos instrumentos y los modelos hidrológicos: evaluación e inventarios hidrológicos; determinación de parámetros hidrológicos; construcción de modelos hidrológicos anexados a un SIG; y construcción de modelos hidrológicos integrados en un SIG. En nuestro caso, estamos ante el tercer tipo, el más frecuente de los cuatro, lo que implica que tanto la herramienta como el modelo mantienen bases de datos separadas que entran en contacto debido a un proceso de intercambio y conversión de la información. Sin embargo, el modelo propuesto va un paso por delante gracias a que el algoritmo ha sido diseñado con una perspectiva geográfica que facilita su implementación en un SIG profesional, es decir, las operaciones lógicas y de cálculo han sido formuladas de tal manera que pueden ser aplicadas directamente a las capas de información, adaptándose sin grandes esfuerzos a las funciones y lenguajes de programación de los SIG.

\subsection{Tratamiento de la precipitación}

En la primera fase del modelo se afronta la problemática de la influencia de las precipitaciones en el comportamiento hidrológico de las ramblas y ríos-rambla. Como ya hemos indicado con anterioridad, en regiones donde son frecuentes situaciones atmosféricas de lluvias intensas la escorrentía tiene carácter torrencial. Asumiendo este contexto, en el que el funcionamiento de los aparatos fluviales está estrechamente vinculado a la aparición de fuertes lluvias concentradas en un corto espacio de tiempo, el estudio de la escorrentía superficial requiere como paso previo ahondar en el comportamiento de las 
precipitaciones. El interés de esta fase reside en averiguar que precipitación máxima se registraría en una cuenca en el caso hipotético de que se produjera el aguacero más desfavorable posible. Esto es, las cantidades máximas registradas en intervalos de tiempo de determinada duración y la frecuencia estimada con la que cabe esperar se reproduzcan o superen esas cantidades (INM, 1999). Combinar esta información con otra relacionada con la duración de las tormentas y la distribución de la lluvia nos permite disponer de series de datos indispensables para la modelación de las precipitaciones. De hecho, la simulación de un episodio de lluvias torrenciales está supeditada a la disponibilidad que tengamos para obtener la estructura de las tormentas.

FIgURA 3. Esquema de la primera fase del modelo hidrológico

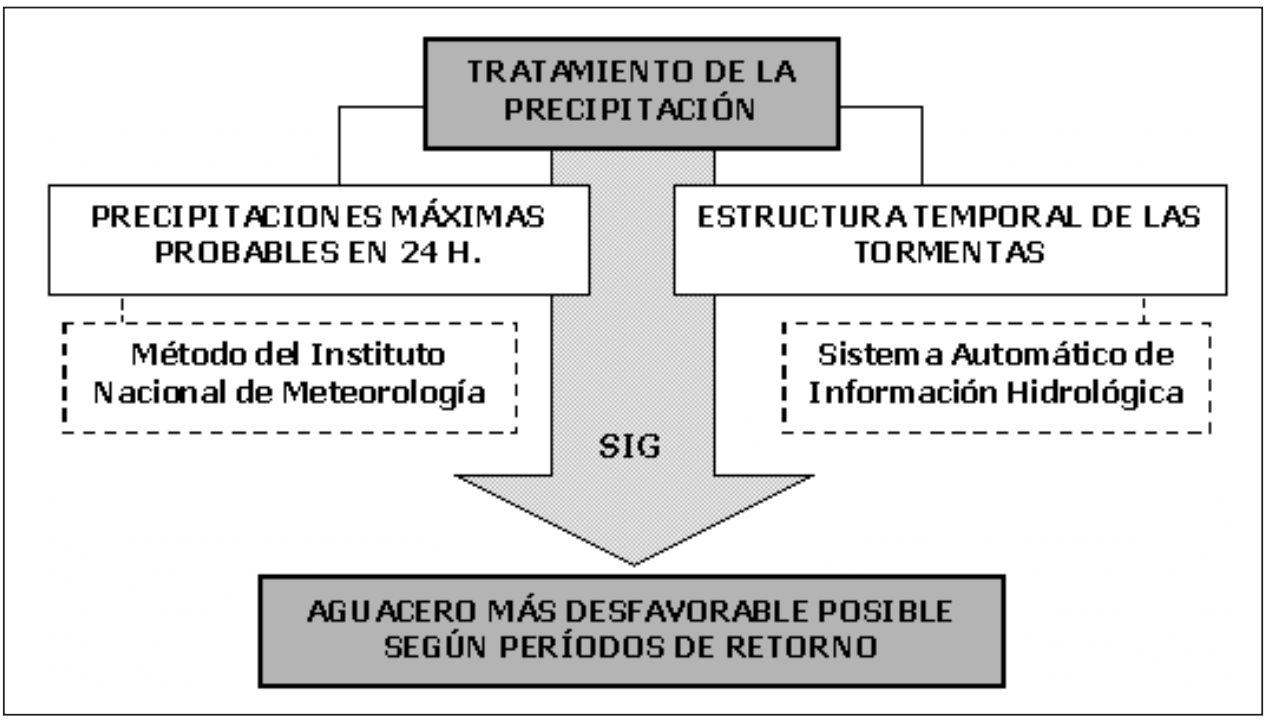

La precipitación máxima probable para un intervalo de tiempo concreto, habitualmente las 24 horas correspondientes al día pluviométrico, se deduce a partir de los registros obtenidos por las estaciones meteorológicas ubicadas cerca y en el interior de la cuenca. Con la ayuda de métodos estadísticos que emplean modelos de leyes de distribución de frecuencia se pueden calcular los valores de precipitación máxima en los puntos que disponen de información pluviométrica. Tomando como referencia estos puntos de muestreo, y gracias a las técnicas de interpolación espacial que incorporan los SIG (p. ej. IDW o kriging), es posible estimar estos valores en aquellos lugares que no cuentan con mediciones directas. Esta predicción matemática dota a la susodicha variable climática de continuidad en el espacio. Para el cálculo de la precipitación máxima hemos evaluado dos métodos, por un lado el de la Dirección General de Carreteras (DGC) del Ministerio de Fomento (1999), y mucho más ajustado a nuestras necesidades, por ser menos conservador, el del Instituto Nacional de Meteorología (INM) del Ministerio de Medio Ambiente (1999). Este método se basa en un ajuste de la ley de distribución de frecuencias para valores extremos de Gumbel y se aplica con resultados satisfactorios a series homogéneas de precipitaciones máximas diarias. La función empleada por el INM (1999), 
pese a contar con algunas limitaciones, hemos observado que se adapta mucho mejor a

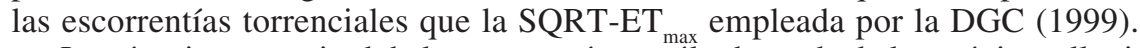

La génesis y magnitud de la escorrentía no sólo depende de las máximas lluvias diarias, por lo que en esta fase también debemos analizar la variabilidad espacial y temporal del agua precipitada. Metodológicamente, esto supone realizar un análisis regional exhaustivo de la estructura de las tormentas en función de las situaciones sinópticas de al menos los últimos 30 años. Se trata de explotar los pluviogramas, siempre y cuando se disponga de ellos, para definir una serie de tormentas «tipo» cuya estructura debe ser característica para cada situación atmosférica. Sus mediciones evidencian la altura real de agua recogida por instante de tiempo durante una tormenta, pero además combinados con las estimaciones sobre la precipitación máxima probable nos permite extrapolar la cantidad de lluvia que se recogería en cada punto de la cuenca suponiendo que se tratase del aguacero más desfavorable posible para un período de retorno dado. Esto se consigue trabajando con los datos de los pluviómetros electrónicos del Sistema Automático de Información Hidrológica (SAIH) de las Confederaciones Hidrológicas, siendo sus registros, continuos y permanentes, la única fuente realmente útil para definir la estructura de las tormentas.

\subsection{Síntesis de la lluvia efectiva}

La construcción teórica del aguacero más desfavorable posible nos ofrece información detallada acerca de la variabilidad de la lluvia en el espacio y en el tiempo, es decir, dónde, cuándo, qué cantidad y con qué intensidad precipita. Comprensiblemente, la segunda fase del modelo consiste en averiguar que fracción de agua de lluvia se convierte en escorrentía, o lo que es lo mismo, se trata de estimar la lluvia efectiva (vid. Figura 4). Los métodos más idóneos para conseguirlo enlazan con la presencia de estaciones de aforo que dispongan de series largas y homogéneas. Sin embargo, esta opción puede ser descartada casi de forma automática debido a que la mayoría de las ramblas y ríos-rambla mediterráneos no están aforados. Así pues, la alternativa radica en emplear un procedimiento que nos permita calcular la lluvia efectiva y los caudales de crecida con arreglo a variables y parámetros hidrológicos conocidos. Los métodos para la obtención del caudal máximo se ajustan a estas necesidades resolviendo con bastante eficacia el problema de la conversión lluviacaudal. De hecho, en la actualidad, este dato se emplea en los Planes Hidrológicos de Cuenca para evaluar el riesgo de inundación, así como en proyectos de infraestructuras viarias para dimensionar los dispositivos de recogida y evacuación de aguas.

El caudal máximo se puede obtener empleando cualquiera de los siguientes cuatro métodos: directos, estadísticos, empíricos e hidrológicos. Los empíricos son los más simples y consisten en aplicar fórmulas matemáticas obtenidas tras largos períodos de observación. El caudal se fija en función de la superficie de la cuenca y una variable característica a escala regional que engloba alguna de sus peculiaridades geográficas (condiciones climáticas, fisiográficas, etc.), posteriormente, el valor obtenido debe ser ajustado mediante estimaciones directas. Los resultados demuestran que la fiabilidad de estos métodos es discutible fuera de las áreas geográficas que les sirvieron de referencia. Sirva como ejemplo un estudio sobre inundaciones realizado en el término municipal de Alicante, en el que aplicando catorce fórmulas para predecir el caudal máximo del barranco de las Ovejas se obtuvo un abanico de valores comprendidos entre 229 y $2.075 \mathrm{~m}^{3} / \mathrm{s}$ (Gil, 1986: 65).

Los métodos hidrológicos, por su parte, han alcanzado un elevado grado de aceptación al contar con ecuaciones más exactas para estimar la lluvia efectiva. Especialmente dos de estos métodos han acumulado, dada su difusión, una dilatada experiencia, nos referimos al racional modificado y al hidrograma unitario. Para esta metodología hemos optado por el 
FIguRA 4. Esquema de la segunda fase del modelo hidrológico

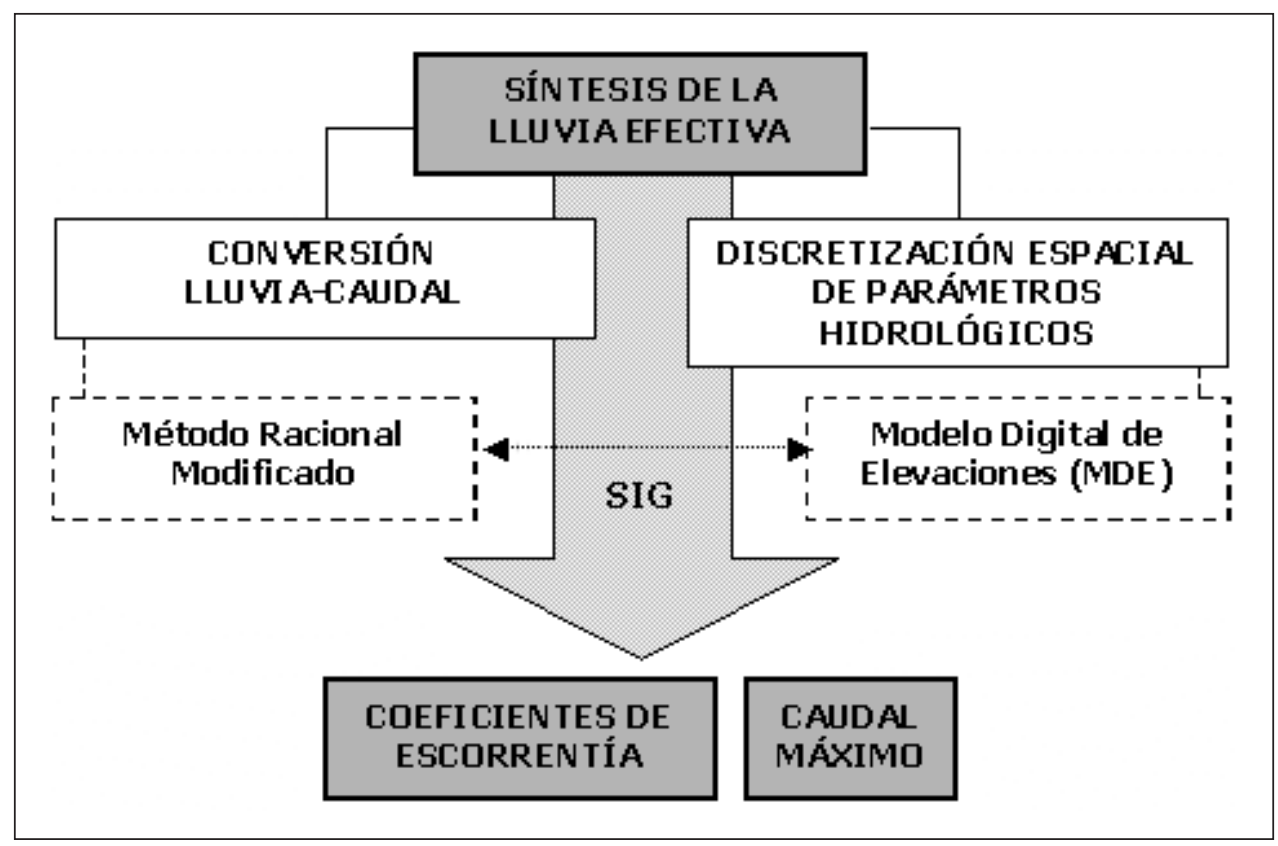

método racional modificado por Témez (1991) al adaptarse con mayor facilidad a las manifestaciones hidrológicas continuas en el espacio y a escalas de trabajo grandes (p. ej. 1:10.000). Estas características, asimismo, se acomodan sin grandes esfuerzos a los modelos de datos de tipo ráster, permitiendo que la información pueda ser trasladada a retículas compuestas por celdas de pequeño tamaño y ser gestionada por un SIG. Otra cuestión que no debemos perder de vista es que este método se apoya en el modelo de infiltración del Soil Conservation Service (1985), lo cual conlleva una serie ventajas relacionadas con su claridad conceptual por el hecho de utilizar un parámetro a modo de coeficiente de escorrentía que es fruto de la combinación de los usos del suelo (incluido el paisaje vegetal) con las características edáficas — se emplea la textura como estimador de la capacidad de retención del agua- y morfológicas del terreno (pendientes topográficas obtenidas de un MDE). La precisión de los coeficientes depende de los datos disponibles sobre las características del suelo y el criterio empleado en la discretización de los usos (p. ej. densidad de las áreas urbanizadas y cobertura de vegetación).

Los métodos hidrológicos, y, en particular, el del número de curva, encuentran en los SIG una herramienta de trabajo muy apropiada para manipular la información espacial del modelo hidrológico. Los SIG están capacitados para interpretar las condiciones geográficas de la cuenca y traducirlas a objetos con propiedades geométricas y atributos. Una vez que las variables hidrológicas son convertidas en capas de información georreferenciada, las funciones de análisis espacial nos permiten realizar operaciones de álgebra de mapas para obtener datos tan relevantes como las pendientes topográficas o los coeficientes de escorrentía. Algunos de estos datos se obtienen a partir de un MDE, es decir, de una 
representación digital fehaciente de la distribución espacial de la altitud de la superficie del terreno (Felicísimo, 1999), siendo los SIG el instrumento más adecuado para crearlos. Precisamente, el MDE ocupa un lugar destacado dentro del modelo hidrológico, no en vano existe una correlación evidente entre la información hipsométrica y el sentido de los flujos, y, en consecuencia, es imprescindible para simular la circulación de la lluvia efectiva.

\subsection{Simulación de la escorrentía superficial}

En las fases anteriores hemos tratado la precipitación y la síntesis de la lluvia efectiva, creando la información necesaria para desarrollar la última fase del modelo hidrológico. En ésta hemos optado por crear un algoritmo capaz de simular la escorrentía superficial atendiendo a un conjunto de reglas estimadas y validadas formal y empíricamente. El algoritmo reproduce el comportamiento de la cuenca durante una precipitación de fuerte intensidad horaria, partiendo del supuesto de que simulando los procesos sencillos de interacción local se puede estimar el proceso global. Desde el punto de vista de la computabilidad, la simulación depende de los elementos del sistema y el grado de resolución considerado. A mayor número de variables o elementos mayor resolución espacial y temporal, obteniéndose, en teoría, unos resultados más verosímiles y próximos a la realidad. Esta deseable aproximación está limitada por la potencia de los ordenadores (memoria y velocidad de procesamiento), de tal manera que forzosamente se deben tomar decisiones que nos permitan obtener unos buenos resultados en un tiempo de cálculo razonable.

Para la simulación se ha realizado una discretización de la información, intrínseca a cualquier proceso informático, dividiendo el tiempo en intervalos y organizando el espacio en una retícula compuesta por celdas. Esto favorece el procesamiento de las relaciones que se dan entre los distintos elementos que componen el ciclo hidrológico durante un aguacero torrencial. Así, la exigencia de una discretización temporal sugiere un algoritmo de simulación iterativo con pasos temporales fijos, mientras que la discretización espacial apunta hacia la conveniencia de emplear datos en formato ráster (si bien esto último no es estrictamente necesario). En nuestro caso una discretización adecuada consiste en tomar intervalos de tiempo del orden del segundo y tamaños de celda del orden del metro. Esto implica importantes requerimientos computacionales, incluso para cuencas pequeñas, siendo imprescindible una gran resolución en los datos de entrada vinculada normalmente a procesos de interpolación espacial.

Los datos necesarios para simular el movimiento y la acumulación de caudales dependen de los niveles de información identificados por el modelo hidrológico. El algoritmo, desarrollado para simular el funcionamiento hidrológico de las ramblas y ríos-rambla mediterráneos, básicamente emplea la estructura espacial y las series temporales del aguacero más desfavorable posible obtenidas en la primera fase, y los coeficientes de escorrentía y el MDE obtenidos en la anterior (vid. Figura 5). Con los coeficientes el programa calcula el umbral de escorrentía y establece las condiciones de humedad en el suelo según lo dispuesto por el método racional modificado y el modelo de infiltración del Soil Conservation Service (1985). Sobre el MDE se aplican las reglas de circulación del agua en el terreno y se obtiene la velocidad de los flujos. El MDE utilizado debe ser «hidrológicamente correcto», es decir, una representación digital de la cuenca cuyas características se ajusten lo máximo posible a la realidad, haciendo hincapié sobre todo en la morfología de los lechos y cárcavas que conducen el agua. Con la información adecuada (curvas de nivel, cotas altimétricas y red hidrológica) un interpolador aconsejable para aplicaciones hidrológicas es recogido por el comando topogrid del SIG ArcInfo de ESRI basado en el progra- 
FIguRA 5. Esquema de la tercera fase del modelo hidrológico

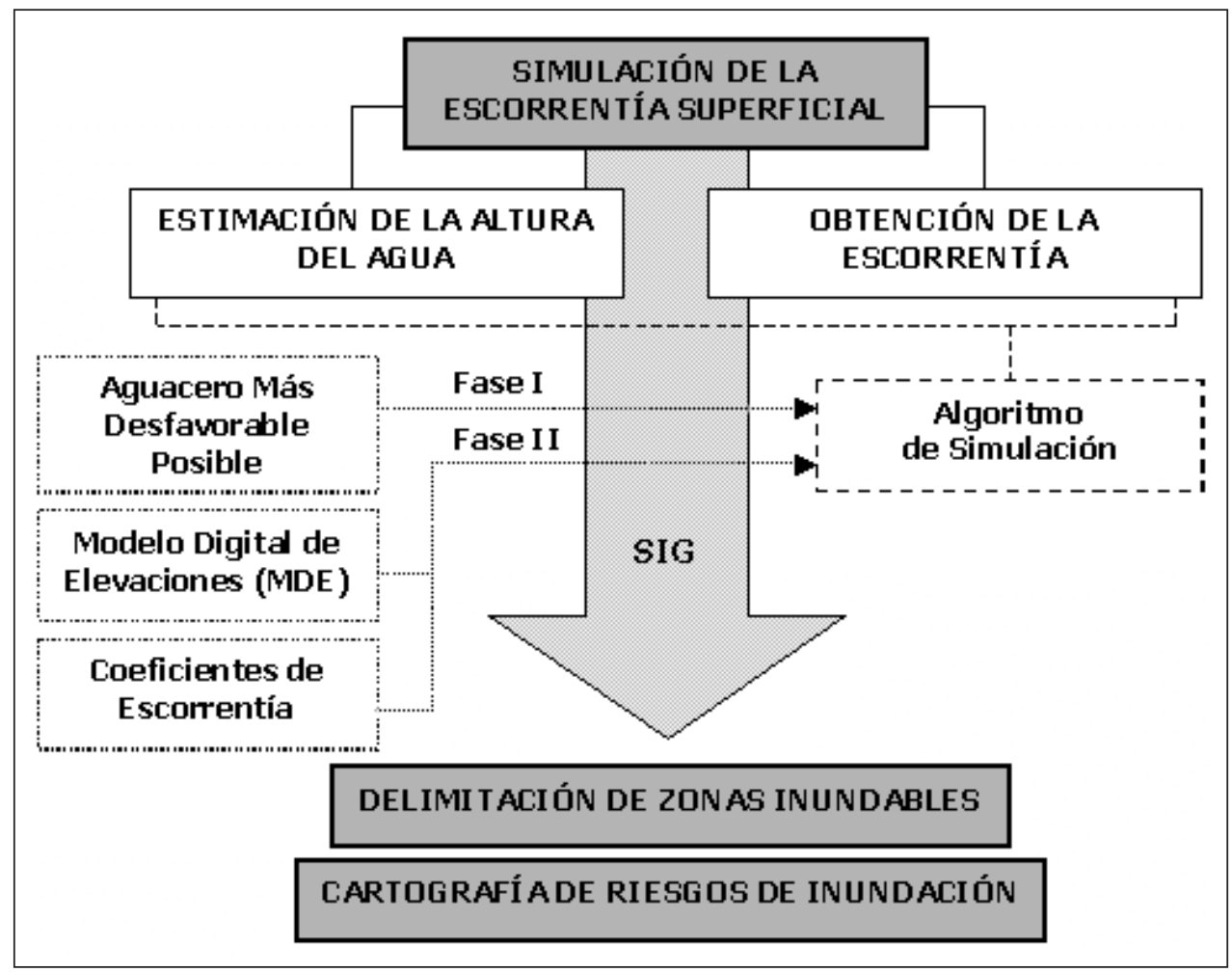

ma ANUDEM de Hutchinson (1988; 1989; 1993). La aportación hídrica del modelo proviene del tratamiento realizado a las precipitaciones. Se toman datos reales para extrapolar su estructura temporal y así obtener la intensidad deseada para la simulación según períodos de retorno. Para estimar la precipitación en toda la cuenca se emplea la técnica determinista del inverso de las distancias al cuadrado (IDW) por ser un interpolador exacto donde los valores máximo y mínimo sólo aparecen en los puntos de muestreo.

El algoritmo de simulación consiste en la aplicación de un procedimiento de cálculo que se repite para cada instante de tiempo, de tal manera que al final de dicho instante se obtienen dos capas de información en formato ráster correspondientes a la cantidad de movimiento y la altura del agua. Este procedimiento considera la situación en el instante previo y las precipitaciones en el actual para estimar el desplazamiento de caudales en la cuenca. En cada iteración se calculan las pendientes topográficas, direcciones, rozamientos, absorciones, flujos y su suma vectorial para obtener el resultado en el instante actual. Estos datos son usados para calcular el siguiente instante. Todas estas interacciones se producen en un intervalo de tiempo lo suficientemente corto como para que puedan realizarse en un espacio geográfico muy localizado, de modo que para cada celda de la retícula sólo es necesario considerar los datos acumulados en su entorno más inmediato sin que se pierda la corrección en los cálculos. Los mapas elaborados constituyen una sucesión que hacen posible construir una animación del proceso de formación de avenidas. Editando 
los resultados en un SIG obtenemos una cartografía detallada de la escorrentía y de sus calados, conocimientos indispensables para delimitar las zonas inundables y realizar la cartografía de riesgos de inundación de un territorio.

\subsection{Funciones del modelo hidrológico}

El modelo hidrológico precisa para su aplicación toda una serie de datos que le son suministrados durante la implementación de las distintas fases que lo componen. Tanto los niveles de información proporcionados por el usuario, como los generados por el propio modelo, están en un formato legible por los SIG, de tal manera que para su manipulación resulta francamente beneficiosa la capacidad analítica y de representación cartográfica que poseen estos instrumentos de trabajo. El modelo nos ofrece múltiples opciones para consultar sus resultados, destacando la posibilidad de hacer valer condiciones relacionadas con la localización geográfica y el horizonte temporal de los datos. Las funciones desarrolladas para estudiar el riesgo de inundación de una cuenca las hemos sintetizado en el siguiente listado, aunque sus posibilidades son ilimitadas dependiendo de las necesidades de cada usuario:

- Permite visualizar el MDE de la cuenca y desplegar los mapas con las variables hidrológicas empleadas por el modelo.

- Delimita cuencas y subcuencas hidrológicas.

- Estima los parámetros hidrológicos de la cuenca y la red de drenaje.

- Muestra la distribución espacial de las precipitaciones en un instante de tiempo dado.

- Establece la dirección preferente de los caudales y las zonas de acumulación de agua.

- Muestra la distribución espacial de la escorrentía y su correspondiente altura del agua en un instante de tiempo dado.

- Muestra la distribución espacial de la altura máxima de agua para un episodio de lluvias determinado.

- Ofrece información sobre el momento en el que se produce la altura máxima de agua en cualquier punto de la cuenca.

- Permite realizar animaciones sobre la evolución en el tiempo y en el espacio de cualquier variable hidrológica (precipitaciones, umbral de escorrentía, grado de saturación del suelo, etc.).

- Permite obtener el calado de una superficie o perfil topográfico definido por el usuario para un instante o período de tiempo dado.

- Facilita la obtención de caudales e identificación del caudal máximo en cada punto de la cuenca.

- Calcula el volumen de agua que ha precipitado y circulado en un espacio concreto de la cuenca durante el evento o en un determinado período de tiempo.

- Calcula los tiempos de inundación. Averigua cuanto tiempo ha estado anegado el terreno bajo unas determinadas condiciones y cuando se produce el caudal máximo.

- Permite relacionar la peligrosidad de la cuenca con los usos del suelo y los instrumentos de ordenación territorial y urbanística para establecer el riesgo de inundación.

- Identifica los espacios que se verían más afectados por un aguacero torrencial. Ayuda a evaluar sus posibles daños económicos y a conocer su distribución espacial. 


\section{Conclusión}

La delimitación de zonas inundables en ramblas y ríos-rambla mediterráneos no puede fundamentarse en criterios subjetivos, ni tampoco en criterios científicos que no consideren como mínimo la altura del agua y el caudal punta según períodos de retorno. Debemos recordar que las cuencas son sistemas abiertos de procesos y respuestas (López Bermúdez, 1992) en cuyo comportamiento participan factores físicos y humanos. Precisamente, la complejidad para estimar la escorrentía superficial estriba en la interdependencia de todos estos factores, y por este motivo, las últimas tendencias en el análisis hidrológico de cuencas se orientan hacia la modelación distribuida de los procesos que en ellas se reproducen. Los modelos hidrológicos distribuidos aportan un esquema teórico que integra de manera lógica los razonamientos empleados para simular la propagación de caudales y niveles de agua en la cuenca durante una precipitación de fuerte intensidad horaria, considerando la variabilidad espacial y temporal de los datos. La utilización de estos modelos implica la aceptación de una nueva filosofía de trabajo que genera muchas reticencias, pues supone abandonan los métodos que se han venido aplicando tradicionalmente (Olivera y Maidment, 1999).

El modelo hidrológico de parámetros distribuidos que proponemos en este artículo ha sido construido con la ayuda de un SIG, lo que mejora considerablemente la gestión y el análisis de la información espacial. La metodología plantea numerosas soluciones para modelar las distintas etapas del ciclo hidrológico en ramblas y ríos-rambla. Con respecto a la variable de las precipitaciones, la circulación hídrica al ser de carácter torrencial, nos obliga a estimar el aguacero más desfavorable posible en función de un análisis regional que considera la precipitación máxima y la estructura de las tormentas. El problema de la conversión lluvia-caudal se abarca desde la perspectiva del caudal máximo, empleando el método racional modificado y el modelo de infiltración del SCS (1985). Por último, la escorrentía se obtiene ejecutando un algoritmo de simulación diseñado específicamente para su aplicación en ámbitos semiáridos. En este procedimiento los SIG se emplean principalmente para construir el MDE y discretizar las variables hidrológicas. Los resultados del modelo nos permiten delimitar con precisión las zonas inundables de ramblas y ríos-rambla mediterráneos, sustentándose en criterios científicos para realizar una cartografía de calidad sobre los riesgos de inundación, una labor de gran responsabilidad que se incrementa cuando adquiere carácter vinculante en los instrumentos de ordenación urbanística y territorial.

\section{Bibliografía}

ANDRYSIAK, P. (2000): Visual floodplain modeling with geographic information systems (GIS). CRWR, University of Texas. Texas (EE.UU.), $274 \mathrm{pp.}$

BESCOS, A. y CAMARASA, A. (2000): «Elaboración de cartografía de zonas inundables. Aplicación al llano de inundación del río Arga (Navarra)», en Serie Geográfica, n 9, pp. 219-236.

BRUNNER, G. (2001): HEC-RAS, River Analysis System. Hydraulic reference manual. HEC. Davis (EE.UU.), $262 \mathrm{pp}$.

CHOUDHRY, S. y MORAD, M. (1998): «GIS errors and surface hydrological modeling: An examination of effects and solutions», en Journal of Survey Engineering, vol. 124, no 3, pp. 134-143.

DIRECCIÓN GENERAL DE CARRETERAS (1999): Máximas lluvias diarias en la España peninsular. Ministerio de Fomento. Madrid, 28 pp.

FELICÍSIMO, A.M. (1999): «La utilización de los Modelos Digitales del Terreno en el estudio del medio físico», en LAÍN, L. (Ed.): Los Sistemas de Información Geográfica en los riesgos naturales y en el medio ambiente. Instituto Tecnológico Geominero de España. Madrid, pp. 127-139. 
GALIANA, T. (1987): Diccionario ilustrado de las ciencias. Larousse. París, 1.563 pp.

GARCIA, S. G. (2002): «A GIS GRASS-embedded decision support framework for flood forecasting», en Proceedings of the Open source GISS-GRASS Users Conference 2002, Trento, 13 pp.

GIL, A. (Dir.) (1986): Inundaciones en la ciudad y término de Alicante. Universidad de Alicante y Ayuntamiento de Alicante. Alicante, 179 pp.

GIL, A. (1993): La propiedad de aguas perennes en el Sureste Ibérico. Universidad de Alicante. Alicante, $191 \mathrm{pp}$.

HUTCHINSON, M. F. (1988): «Calculation of hydrologically sound digital elevation models», en Third International Symposium on Spatial Data Handling. International Geographical Union. Sydney.

HUTCHINSON, M. F. (1989): «A new procedure for gridding elevation and stream line data with automatic removal of spurious pits», en Journal of Hydrology, vol. 106, pp. 211-232.

HUTCHINSON, M. F. (1993): «Development of a continent-wide DEM with applications to terrain and climate analysis», en GOODCHILD, M. F. (Ed.): Environmental modeling with GIS, Oxford University Press, Nueva York, pp. 392-399.

HYDROLOGIC ENGINEERING CENTER (2001): Differences between HEC-HMS and HEC-1. HEC. Davis (EE.UU.).

INSTITUTO NACIONAL DE METEOROLOGÍA (1999): Las precipitaciones máximas en 24 horas y sus períodos de retorno en España. Un estudio por regiones. Introducción y Metodología. Ministerio de Medio Ambiente. Madrid, 18 pp.

LÓPEZ ANDRÉS, L. (1997): Manual de hidráulica. Universidad de Alicante. Alicante, 414 pp.

LÓPEZ BERMÚDEZ, F. (1992): «Geomorfología», en LÓPEZ BERMÚDEZ, F., RUBIO, J. M. y CUADRAT, J. M. (Eds.): Geografía física. Cátedra. Madrid, pp. 19-227.

MAIDMENT, D. (1993): «GIS and hydrologic modeling», en GOODCHILD, M., PARKS, B. y STEYAERT, L. (Eds.): Environmental Modeling with GIS. Oxford University Press. New York, pp. 147-167.

MAIDMENT, D. y DJOKIC, D. (Eds.) (2000): Hydrologic and hydraulic modeling support with Geographic Information Systems. ESRI. Redlands (EE.UU.), 216 pp.

MARTÍNEZ, V, DAL-RÉ, R., GARCÍA, A. I., AYUGA, F. (2000): «Modelación distribuida de la escorrentía superficial en pequeñas cuencas mediante SIG. Evaluación experimental», en Ingeniería Civil, no 117, pp. 49-58.

MATEU, J. F. (1989): «Ríos y ramblas mediterráneos», en GIL, A. y MORALES, A. (Dirs.): Avenidas fluviales e inundaciones en la cuenca del Mediterráneo. Instituto Universitario de Geografía de la Universidad de Alicante y Caja de Ahorros del Mediterráneo. Alicante, pp. 133150.

MORAD, M. y TRIVIÑO, A. (2001): «Sistemas de Información Geográfica y modelizaciones hidrológicas: Una aproximación a las ventajas y dificultades de su aplicación», en Boletín de la Asociación de Geógrafos Españoles, n 31, pp. 23-46.

NATIONAL CENTER FOR GEOGRAPHIC INFORMATION AND ANALYSIS (1990): NCGIA Core Curriculum. University of California. Santa Bárbara (EE.UU.).

OLIVERA, F. y MAIDMENT, D. (1999): System of GIS-Based hydrologic and hydraulic applications for highway engineering: Summary report. University of Texas. Austin (EE.UU.), 28 pp.

SOIL CONSERVATION SERVICE (1985): National engineering handbook. Section 4: "Hydrology». United States Government Printing Office. Washington D.C. (EE.UU.).

TATE, E. (1999): Floodplain mapping using HEC-RAS and ArcView GIS. CRWR, University of Texas. Texas (EE.UU.), 223 pp.

TÉMEZ, J. R. (1991): «Extended and improved rational method. Version of the highways administration of Spain», en XXIV Congreso Internacional de la IAHR. International Association of Hydrological Resources. Madrid.

TRIVIÑO, A. (2002): Los sistemas de indicadores en la planificación del turismo. Propuesta para el análisis del riesgo de inundación en el litoral del Bajo Segura. Memoria de Licenciatura (inédita), Universidad de Alicante. Alicante, $330 \mathrm{pp}$. 
